\title{
UPPER BOUNDS FOR THE STRONG CHROMATIC INDEX OF HALIN GRAPHS
}

\author{
ZIYU HU \\ Department of Mathematical Sciences \\ Florida Atlantic University \\ e-mail: azuth.hu@gmail.com \\ KO-WEI LIH \\ Institute of Mathematics, Academia Sinica \\ e-mail: makwlih@sinica.edu.tw \\ AND \\ DAPHNe DeR-Fen LiU ${ }^{1}$ \\ Department of Mathematics \\ California State University Los Angeles \\ e-mail: dliu@calstatela.edu
}

\begin{abstract}
Keywords: strong edge-coloring, strong chromatic index, Halin graphs.
\end{abstract}

2010 Mathematics Subject Classification: 05C15.

The strong chromatic index of a graph $G$, denoted by $\chi_{s}^{\prime}(G)$, is the minimum number of vertex induced matchings needed to partition the edge set of $G$. Let $T$ be a tree without vertices of degree 2 and have at least one vertex of degree greater than 2. We construct a Halin graph $G$ by drawing $T$ on the plane and then drawing a cycle $C$ connecting all its leaves in such a way that $C$ forms the boundary of the unbounded face. We call $T$ the characteristic tree of $G$. Let $G$ denote a Halin graph with maximum degree $\Delta$ and characteristic tree $T$. We prove that $\chi_{s}^{\prime}(G) \leqslant 2 \Delta+1$ when $\Delta \geqslant 4$. In addition, we show that if $\Delta=4$ and $G$ is not a wheel, then $\chi_{s}^{\prime}(G) \leqslant \chi_{s}^{\prime}(T)+2$. A similar result for $\Delta=3$ was established by Lih and Liu [21].

\footnotetext{
${ }^{1}$ Corresponding author. Supported in part by grants NASA MIRO NX15AQ06A and NSF DMS 1600778.
} 


\section{REFERENCES}

[1] L.D. Andersen, The strong chromatic index of a cubic graph is at most 10, Discrete Math. 108 (1992) 231-252. doi:10.1016/0012-365X(92)90678-9

[2] J. Bensmail, A. Harutyunyan, H. Hocquard and P. Valicov, Strong edge-coloring of sparse planar graphs, Discrete Appl. Math. 179 (2014) 229-234. doi:10.1016/j.dam.2014.07.006

[3] O.V. Borodin and A.O. Ivanova, Precise upper bound for the strong edge chromatic number of sparse planar graphs, Discuss. Math. Graph Theory 33 (2013) 759-770. doi:10.7151/dmgt.1708

[4] H. Bruhn and F. Joos, A stronger bound for the strong chromatic index, Electron. Notes Discrete Math. 49 (2015) 277-284.

doi:10.1016/j.endm.2015.06.038

[5] G.J. Chang and G.-H. Duh, On the precise value of the strong chromatic-index of a planar graph with a large girth.

arXiv: 1508.03052

[6] G.J. Chang and D. Liu, Strong edge-coloring for cubic Halin graphs, Discrete Math. $312(2012)$ 1468-1475. doi:10.1016/j.disc.2012.01.014

[7] G.J. Chang, M. Montassier, A. Pêcher and A. Raspaud, Strong chromatic index of planar graphs with large girth, Discuss. Math. Graph Theory 34 (2014) 723-733. doi:10.7151/dmgt.1763

[8] D.W. Cranston, Strong edge-coloring of graphs with maximum degree 4 using 22 colors, Discrete Math. 306 (2006) 2772-2778. doi:10.1016/j.disc.2006.03.053

[9] P. DeOrsey, J. Diemunsch, M. Ferrara, N. Graber, S.G. Hartke, S. Jahanbekam, B. Lidicky, L.L. Nelsen, D. Stolee and E. Sullivan, On the strong chromatic index of sparse graphs.

arXiv: 1508.03515

[10] P. Erdős, Problems and results in combinatorial analysis and graph theory, Discrete Math. 72 (1988) 81-92. doi:10.1016/0012-365X(88)90196-3

[11] P. Erdős and J. Nešetřil, Problem, in: Irregularities of Partitions, G. Halász and V.T. Sós (Eds.) (Springer, Berlin, 1989) 162-163.

[12] R.J. Faudree, R.H. Schelp, A. Gyárfás and Zs. Tuza, The strong chromatic index of graphs, Ars Combin. 29B (1990) 205-211.

[13] J.L. Fouquet and J. Jolivet, Strong edge-coloring of graphs and applications to multi$k$-gons, Ars Combin. 16A (1983) 141-150.

[14] J.L. Fouquet and J. Jolivet, Strong edge-coloring of cubic planar graphs, Progress in Graph Theory in: J.A. Bondy and U.S.R. Murty (Ed(s)), (Academic Press, Toronto, 1984) 247-264. 
[15] M.C. Golumbic and M. Lewenstein, New results on induced matchings, Discrete Appl. Math. 101 (2000) 157-165. doi:10.1016/S0166-218X(99)00194-8

[16] H. Hocquard, M. Montassier, A. Raspaud and P. Valicov, On strong edge-colouring of subcubic graphs, Discrete Appl. Math. 161 (2013) 2467-2479. doi:10.1016/j.dam.2013.05.021

[17] P. Horák, The strong chromatic index of graphs with maximum degree four, in: R. Bodendiek and K. Wagner $(\mathrm{Ed}(\mathrm{s}))$, Contemporary Methods in Graph Theory (BI Wissenschaft-Verlag, Mannheim, 1990) 399-403.

[18] P. Horák, H. Qing and W.T. Trotter, Induced matchings in cubic graphs, J. Graph Theory 17 (1993) 151-160. doi:10.1002/jgt.3190170204

[19] D. Hudák, B. Lužar, R. Soták and R. Škrekovski, Strong edge-coloring of planar graphs, Discrete Math. 324 (2014) 41-49. doi:10.1016/j.disc.2014.02.002

[20] H.-H. Lai, K.-W. Lih and P.-Y. Tsai, The strong chromatic index of Halin graphs, Discrete Math. 312 (2012) 1536-1541. doi:10.1016/j.disc.2011.09.016

[21] K.-W. Lih and D.D.-F. Liu, On the strong chromatic index of cubic Halin graphs, Appl. Math. Lett. 25 (2012) 898-901. doi:10.1016/j.aml.2011.10.046

[22] M. Molloy and B. Reed, A bound on the strong chromatic index of a graph, J. Combin. Theory Ser. B 69 (1997) 103-109. doi:10.1006/jctb.1997.1724

[23] W.C. Shiu, P.C.B. Lam and W.K. Tam, On strong chromatic index of Halin graphs, J. Combin. Math. Combin. Comput. 57 (2006) 211-222.

[24] W.C. Shiu and W.K. Tam, The strong chromatic index of complete cubic Halin graphs, Appl. Math. Lett. 22 (2009) 754-758. doi:10.1016/j.aml.2008.08.019

[25] J. Wu and W. Lin, The strong chromatic index of a class of graphs, Discrete Math. 308 (2008) 6254-6261. doi:10.1016/j.disc.2007.11.051

Received 17 September 2015

Revised 30 August 2016

Accepted 30 August 2016 